\title{
Using Wireless Communication to Improve Road Safety and Quality of Service at Road Construction Work Sites (Poster)
}

\author{
David Rylander \\ Volvo Construction Equipment \\ Eskilstuna, Sweden \\ David.rylander@volvo.com
}

\author{
Jakob Axelsson \\ School of Innovation, Design and Engineering \\ Mälardalen University \\ Västerås, Sweden \\ jakob.axelsson@mdh.se
}

\begin{abstract}
This paper investigates how wireless communication and vehicular ad hoc network (VANET) based technologies can be applied in relation to road construction work sites to improve safety and increase efficiency and sustainability. It discusses how to design the flow of data for a number of functions and how to operate the functions at the work site even at low VANET penetration rates. The applications described identify the potential in increased productivity at a road construction site and how increased visibility of site activities impact on traffic and improved road safety. It also highlights important implementation considerations and the need of availability and utilization of open standards for this purpose.
\end{abstract}

Keywords- Wireless Communication, Cooperative Systems, VANET, V2X, M2X, Vehicular Communication, Road construction work site, ETSI-G5.

\section{INTRODUCTION}

In recent years there has been an increased attention on using wireless communication to improve e.g., the degree of automation, active safety, energy efficiency and engine control, supply chain visibility and traffic management within the domain of road transports. The results from these activities have advanced the development of wireless vehicle-to-vehicle (V2V) and vehicle-to-infrastructure (V2I) communication. The needs of such V2V and V2I (or, for short, V2X) communication based services has resulted in the development of Vehicular Ad Hoc Network (VANET) technologies.

The aim for using VANET for road vehicles is to create a reliable electronic digital awareness about the vehicle's surroundings. The main technical concept is that all vehicles periodically broadcast a set of attributes (e.g., speed, position, direction) to all other connected vehicles within range. This information is used by the receiver to increase its awareness of each surrounding vehicle's status, information and predicted future situation. This awareness within each vehicle can then be used e.g., to optimize traffic flow, decrease fuel consumption and prevent accidents.

An area within road transports that has not been much investigated with respect to road vehicle based wireless communication, is road construction work site operations. The purpose of this paper is to open up this area and highlight some of the needs and application potential.

One of the main challenges when commercializing VANET based offers for road vehicles is how to introduce benefits even for the first users. With what and whom will these first equipped vehicles communicate and what data will be exchanged? Many applications for road vehicles will in fact be useless unless a substantial penetration rate is reached. In this paper, we will show that the technology actually provides a value for road construction sites even at a low penetration rate.

The rest of the paper is structured as follows: In the next section, an overview of some related research is given, and then three applications are presented where wireless communication is used at road construction works. In the following section, implementation considerations peculiar to these applications are discussed, and finally the conclusions are summarized together with indications of future research in the area.

\section{RELATED WORK}

Within the recent research on VANET and wireless vehicle communication the focus has been on the ITS station [2] e.g., communication architecture and related functions [3], performance [4] and vehicle centric applications. The VANET research related to road construction work sites has been focusing on the applications related to road construction sites from a road user perspective. These applications have been developed based on road works information as a warning or trigger for actions. This concept has been developed within multiple projects such as EU-CVIS [5], EU-SafeSpot [6], EUCOSMO [7] and EU-CO-Cities [8]. The result from these efforts includes the basic information exchange between ITS stations such as vehicles and road construction sites. This has also been developed, considered and included within wireless message standardization in ETSI-ITS [9] and in ISO-TPEG [10].

Another research area of road construction is planning, design and information management. Here research concludes and identifies different strategies and processes [11] for how 
information management should be handled efficiently. The utilization of wireless communication for specific tasks [12] have been investigated and the desired position and need of information described [13]. But this research has not been covering how the emerging VANET technologies and standardized protocols can be utilized. It has also been identified that a system solution needs to be technically, economically and operationally feasible [11] which implies that a solution needs to be interoperable and widely used for it to be useful.

Since the focus within these related research and standardization activities has not been on how the actual layout and wireless functions at the road construction work site should be designed, utilized and technically solved in an optimal way, considering functionality, interoperability and quality of service. The contribution of this paper is to open up the area of road construction improvements using wireless technologies describing the needs and propose solutions for this purpose. More in detail the paper describe how emerging wireless communication technologies (e.g., VANET) and standards can be used to improve a number of applications and how to operate the functions at the construction work site

\section{WIRELESS COMMUNICATION BASED APPLICATIONS WITHIN ROAD CONSTRUCTION}

There is a large number of areas within road construction where the use of wireless communication can have benefits. In this chapter we will discuss three such areas that are particularly promising.

Since road work and road constructions usually block parts of the road, there are often queues formed and increased travel times as a consequence. Another issue is that road works often cause accidents. According to [14], road works increase the accident rate with $21.4 \%$ for freeways in the US. One of the main reasons is lack of accurate information and awareness of the approaching drivers, e.g., about the actual presence of the road work, the layout of the road work and how the road accessibility and throughput is affected as well as how the temporary speed limit is defined for the area. To improve the current situation there is a need for ICT infrastructure to detect and notify road users accurately and in real-time about e.g., congestions, queues, travel times, current speed limit or accidents.

In addition to these needs it cannot necessarily be assumed that the road construction work areasare geographically positioned where the information and communication technology (ICT) infrastructure such as cellular communication or fixed queue and incident detection systems has optimal support and coverage.

When the required coverage cannot be guaranteed to be as mobile as the need of road infrastructure improvements and related road work activities, there is a need of having new moveable and interoperable ICT-solutions that detect these events and notify road users in an improved, accurate and precise way related to road construction site activities.

Another emerging area for wireless communication is road work site production control and synchronization of vehicles and machines working at and towards the site. Compared to other production processes such as factories there are no universal site status and control system available today.

This chapter discusses and proposes solutions in terms of functional flows to the following identified wireless communication and VANET based applications related to road construction work sites:

- Traffic incident detection

- Road work status information dissemination

- Road work operation control

\section{A. Traffic incident detection}

At road construction sites there is a need to warn and notify road users about the incident and related traffic situations. To be able to do so there is a need of reliable, accurate detection and identification of such incidents. Today there are several fixed solutions to this but few that are connected to the road works sites. However, emerging technologies such as VANET could be used to improve the situation.

The basic function of the standard VANET/ETSI ITS-G5 [15] is that the vehicles that are equipped broadcast their speed, position and direction to all nodes within range [9]. If there would be a receiver for this data at each road construction work site, this information would be enough to be able to detect travel times and potential queues even if not all road vehicles are equipped. The reason is that it can be assumed that all vehicles in a queue have the same approximate travel time for the specific road stretch. When one or more vehicles transmit its data with a certain travel time, it can be applied also on the whole road stretch affected as an approximated value. For this reason the application adds value even with a low penetration rate of VANET in vehicles, even though the value and accuracy increases with an increased penetration rate of vehicles. This is important, since the introduction of VANET technology will be gradual starting from a low level.

The main flow of events for the traffic incident detection application is as follows (see Figure 1):

1. VANET equipped vehicle approaches road construction worksite.

2. Approaching vehicle decrease speed

3. Machine at worksite receives vehicle speed and position and determines if there is a queue and/or increase in travel time

4. If queue or travel time increase is detected, machine notifies related traffic management function through long range communication, e.g., cellular or satellite, about the current conditions for further actions to take (e.g., send RDS-TMC for improved navigation and route planning services) 


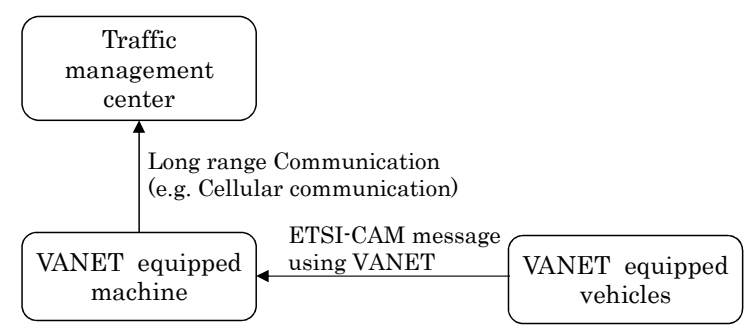

Figure 1. Functional flow of events for detection application

\section{B. Road work information dissemination}

Today the information about road works is often accessed from the local road operator and its traffic management functions. For dissemination purposes the information is often incorporated into broadcast of Traffic Message Channel (TMC) messages such as RDS-TMC and DAB. These TMC messages are e.g., received by navigation systems used for related notifications to the driver and can be utilized for efficient routing and navigation. This is beneficial since road works often cause congestions, queues and related increase of travel times and fuel consumption. Another way of disseminating the data is to incorporate the information into other services where the data can be merged and processed for added value of that specific service e.g., internet based off-board route planning and navigation. Unfortunately this information is not totally reliable or accurate in position, description or in time, especially not if it would be used for safety critical systems.

The lack of reliability and accuracy is mainly due to the source and the message format used. The source of road work operation information generally comes from the road construction entrepreneurs operating the work. Taking Sweden as an example, the entrepreneur need to announce well in advance (three weeks) before starting the operation what road stretch will be affected and the start/end date and time. This is not always the exact same stretch and time when the work starts, which in some cases causes faulty TMC notifications and warnings. In any case, the TMC message format does not state how traffic is affected in terms of queues and travel times, and not how the actual road stretch is affected geographically with suitable position accuracy for active safety functionality.

The VANET technology discussed above for detecting traffic situations can also be used to disseminate safety critical messages to road users through the very same technical platform. This approach allows for a direct open channel towards the road users with minimum amount of latencies and dependencies in terms of systems and nodes in between sender and receiver. This is naturally a suitable solution for safety critical systems. The information detected in terms of approaching vehicles can in some cases also be used to warn workers at the site about e.g., approaching high speed vehicles or other type of specific vehicles such as dangerous goods transports which may require special attention.

In addition this reliable and accurate detected data through VANET can be used to disseminate the very same information through existing channels e.g., TMC. The application would be facilitated already with a small number of VANET equipped vehicles for detection and acquisition. But the approach would add value in terms of increased reliability and accuracy of already available services for TMC, variable message signs and other means of disseminating traffic incidents such as congestions, travel times and accidents in relation to road works.

The main flow of events for the road work notification and warnings dissemination application is as follows (see also Figure 2):

1. Road work machines directly inform approaching vehicles (M2V) about its presence (e.g., road worksite area) and queue/travel time situation. This information would be received by the user with minimal latency and without several systems and components in between the source and user. This is the optimal solution for safety critical systems and actions.

2. Approaching vehicles receive the message and can take actions, e.g., warn/notify driver about the situation.

3. Road work machines inform local traffic management functions about the current status, e.g., operational site/road work start/end date and time as well as area and current queue/travel time situation.

4. Local traffic management functions (in some cases through service providers) provide services such as radio broadcast (e.g., RDS-TMC) or cellular IP-based solutions for informing road users about the situation and recommended actions.



Figure 2. Functional picture of notification and warning dissemination application

\section{Road work operation control}

At a road work there are usually several machines operating. The machines are all designed to perform individual specialized tasks such as digging, paving, asphalt laying, compacting and transporting. These machines work in specific processes (see Figure 3) and in a defined sequence. 




Figure 3. Processes involved in road construction [1]

Comparing this process with other production processes such as factories the main difference is that for road construction there are no interoperable digital process control systems. The operator of each task is very rarely connected to the other tasks and sub processes digitally. The awareness and synchronization of the related tasks is done orally and visually between the workers. Since a site often involves several contractors and many workers the communication between these tasks and processes is very limited and takes a lot of time. Due to this there is a need of digital, interoperable and harmonized communication of the current operational work status. This includes the location of each machine and what it is doing, for the other related tasks to adapt towards. As an example there is a need for compactors (when more than one at a site) to be synchronized for optimal area compacting coverage. Positions, heading and speed would be enough to generate path history and trajectories to use for compaction optimization, assuming that the compactors are within communication range. Another example would be for asphalt layers to communicate with its asphalt supplier/transports to be able to synchronize mass need and supply. Both of these examples are expected to increase operational uptime and productivity at a site. Naturally the application assumes that the machines have the awareness of its task and mission. This can be configured either by the operators directly or by a central task and process management system (site coordinator). Additional functionality would be to monitor the site progress and to be able to assign tasks to machines and manage resources towards more demanding tasks.

The main flow of events for the road work control application is as follows (see Figure 4):

1. VANET equipped machine broadcast activity (machine type, machine task, operational status) and basic position data (position, speed, and heading)

2. Other VANET equipped machines can adapt related activity and task towards this information and broadcast back the same information about its own status (machine type, machine task, operational status, position, speed, and heading). Using this information the machine and operator can adapt its activity pace and plan for the future activity needed.
3. Additionally the site coordinator/operator can monitor the progress, assign tasks and change priorities for the tasks and missions dedicated to the machines required at the site. This can be done both utilizing a VANET or other, e.g., Internet Protocol (IP) based technologies.

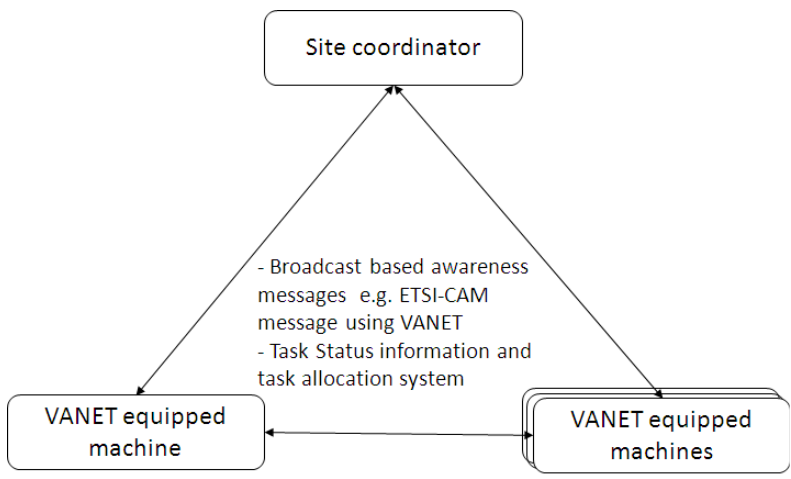

Figure 4. Functional picture of the communication required for the operation control application

\section{IMPLEMENTATION CONSIDERATIONS}

The technology for sending and receiving VANET messages has been proven functional and trialed in operation at multiple projects and occasions. It is mature and has a reasonable range even though the range is heavily affected by lack in line of sight [16]. There is also another relevant lack identified regarding packet loss with the currently used MAC method [4, 17], but these issues have very little effect on the type of applications described in this paper since the information is not time critical in terms of the lack of performance. What is prominent is instead the need of a reliable power source at all time. It would also be of benefit if the antenna is placed at a high location since the $5.9 \mathrm{GHz}$ frequency band is easily obstructed.

The approach we propose is that the road construction machines would be the center node of incident detection, acquisition and dissemination of traffic incidents due to road works. It would also be of great benefit for the operational control of a site. At a general road construction jobsite there are machines present at all times. Machines have a reliable power source, they are mobile and are placed at the road work since it is their operational jobsite. In addition most machines used, such as excavators, wheel loaders, pavers, graders and compactors are large machines and in most cases have a height higher than cars. This result in good conditions for unobstructed communication range (line of sight) which is optimal for the frequency planned. When the machines are connected with reliable and accurate global positioning solutions, they can also determine the actual current site location and how its predicted layout affects the traffic flow. The actual traffic flow can then be detected and monitored by the machines through VANET messages from passing equipped vehicles. In addition, the machines can inform about the road work affected area and when it is operational and finalized, simply by its appearance. 
The machines do not only have the technical advantages or location, height (range) and power supply accessibility. They also have a direct business relation with the operator of theroad. This simplifies the process of agreeing protocol and message reception of, e.g., traffic queue and travel time information to be further aggregated in the system, but also how requirements on equipment and functionality of the machines operating at the site are defined.

A general work site often has machines from different brands and from different sub-contractors. The implementation of a system solution that controls and monitors the progress at a site involving the full flow of missions, tasks and machines, would strongly benefit from an open standard implementation. Naturally the same approach is required to be able to communicate with the surrounding traffic. A closed local system solution will not be interoperable with the road vehicles passing the site and would not be able to acquire the needed real-time data. Deploying a solution then requires that open standards exist and are utilized by the system suppliers. For the control and synchronization of machines as well as the machine positions and movement monitoring the VANET approach utilizing e.g., CAM-messages would be enough for this purpose. But for the task allocation as well as progress monitoring and control there would be a need for a global standard protocol adapted towards road construction.

\section{CONCLUSIONS AND FUTURE WORK}

From the discussion within this paper the following conclusions can be made:

1. Wireless communication and VANET can facilitate improved road safety, and quality of service of traffic data such as congestion and queue information for improved route advice and navigation solutions.

2. To be able to have full control and monitoring of the site and its surrounding traffic requires availability and utilization of open interoperable communication standards for VANET and wireless communication.

3. Wireless communication and VANET has a great potential in increasing the productivity and operational control and awareness at a site.

4. Road construction site improvements based on VANET can be among the first applications for market introduction. The applications have the potential of improving road safety and quality of service even with low penetration rate and amount of equipped vehicles.

5. Road construction machines are suitable objects to place wireless communication and VANET equipment on. This is the case due to its power supply, location at the site and natural height which provide good conditions for acceptable communication coverage and range, for e.g., collecting, analyzing and disseminating traffic related information connected to road construction sites.

6. There is a need to review and update the standards for supporting communication related to road construction work sites.
In the future, there is a need for more research activities, simulations and field operational tests where road construction site improvements are fully considered and assessed, including equipped road construction machines. Many more applications than the three examples presented in this paper will also be detailed.

\section{ACKNOWLEDGMENT}

The authors would like to thank Mr. Bo Bjerkeholt at Trafikverket (The Swedish Transport Administration) for valuable assistants and knowledge about Swedish procedures related to road construction work sites.

\section{REFERENCES}

[1] Volvo CE. (2012, October 1). Volvo Corporate Presentation 2012. Available: http://www.volvoce.com

[2] ETSI, "EN 302665 Intelligent Transport Systems (ITS); Communications Architecture," ed, 2010.

[3] A. Böhm and M. Jonsson, "Real-Time Communication Support for Cooperative, Infrastructure-Based Traffic Safety Applications," International Journal of Vehicular Technology, vol. 2011, 2011.

[4] B. Kloiber, T. Strang, M. Rockl, and F. de Ponte-Muller, "Performance of CAM based safety applications using ITS-G5A MAC in high dense scenarios," in Intelligent Vehicles Symposium (IV), 2011 IEEE, 2011, pp. 654-660.

[5] CVIS. (2012, October 1). Available: http://www.cvisproject.org

[6] SafeSpot. (2012, October 1). Available: http://www.safespot-eu.org/

[7] COSMO. (2012, October 1). Available: http://www.cosmo-project.eu/

[8] CoCities. (2012, October 1). Available: http://www.co-cities.eu/

[9] ETSI, "TR 102638 Intelligent Transport Systems (ITS); Vehicular Communications; Basic Set of Applications; Definitions," ed, 2009.

[10] CEN/ISO, "TS 18234-4:2007 "Traffic and Travel Information (TTI) TTI via Transport Expert Group (TPEG) data-streams- Part 3:Road Traffic Message (RTM) application," 2007.

[11] S. L. Bowden, Dorr, A., Thorpe, A., Anumba, C.J., "Mapping site processes for the introduction of mobile IT," Proceedings of the 5th European Conference on Product and Process Modelling in the Building and Construction Industry, Istanbul, Turkey, (2004.

[12] F. Peyret, J. Jurasz, A. Carrel, E. Zekri, and B. Gorham, "The Computer Integrated Road Construction project," Automation in Construction, vol. 9, pp. 447-461, 2000.

[13] Y. Chen and J. M. Kamara, "Using mobile computing for construction site information management," Engineering, Construction and Architectural Management, vol. 15, p. 13, 2008.

[14] A. J. Khattak, "Effects of work zone presence on injury and non-injury crashes," Accident analysis and prevention, vol. 34, p. 19, 2002.

[15] ETSI, "ES 202 663, "Intelligent Transport Systems; European profile standard on the physical and medium access layer of $5 \mathrm{GHz}$ ITS"," ed, 2009.

[16] A. Böhm, K. Lidstrom, M. Jonsson, and T. Larsson, "Evaluating CALM M5-based vehicle-to-vehicle communication in various road settings through field trials," in Local Computer Networks (LCN), 2010 IEEE 35th Conference on, 2010, pp. 613-620.

[17] K. Bilstrup, E. Uhlemann, E. G. Ström, and U. Bilstrup, "On the ability of the 802.11p MAC method and STDMA to support real-time vehicleto-vehicle communication," EURASIP J. Wirel. Commun. Netw., vol. 2009, pp. 1-13, 2009. 